\title{
ACESSO À CRECHE E ESTADO NUTRICIONAL DAS CRIANÇAS BRASILEIRAS: DIFERENÇAS REGIONAIS, POR FAIXA ETÁRIA E CLASSES DE RENDA'
}

\section{ACCESS TO DAY-CARE CENTERS AND THE NUTRITIONAL STATUS OF BRAZILIAN CHILDREN: REGIONAL DIFFERENCES BY AGE GROUP AND INCOME CLASS}

\author{
Marina Vieira da SILVA ${ }^{2}$ \\ Ana Maria Holland OMETTO2 \\ Maria Cristina Ortiz FURTUOSO² \\ Maria Angélica Penati PIPITONE² \\ Gilma Lucazechi STURION²
}

\section{RESUMO}

O trabalho visa descrever o acesso das crianças brasileiras às creches nas diferentes macro-regiões, centralizando a atenção no estado nutricional e, também, nas classes de renda. A base de dados adotada é constituída pelas informações individuais das crianças $(n=10667)$ menores de seis anos, integrantes da Pesquisa Nacional sobre Saúde e Nutrição, realizada em 1989. De forma geral os resultados demonstram que os programas de creches favorecem as crianças mais velhas (com dois anos ou mais). Verificam-se que apenas $8,49 \%$ das crianças, com renda domiciliar per capita menor do que US\$40.00 freqüentam creche. No grupo com rendimento per capita pelo menos igual a US\$80.00, aquela proporção alcança 28,37\%. A menor cobertura das creches foi observada na Região Centro-Oeste (apenas 10,94\% das crianças freqüentavam a creche). Quando se analisa o estado nutricional das crianças observa-se, de forma sistemática, maiores proporções de crianças com escore-Z de altura para idade <-2 entre aqueles que não freqüentam creche.

Termos de indexação: antropometria, cuidado da criança, estado nutricional, nutrição infantil, política de saúde, saúde infantil.

\begin{abstract}
The aim of this paper is to describe the access of Brazilian children to day-care centers in different macro-regions, focusing on their nutritional status and considering their income level. The data used was individual information of under six-year-old children $(n=10,667)$ from the Health and Nutrition National Research, carried out in 1989. The overall results showed that day-care center programs favor older children (two-year-old and over). Among children with per capita household income below US $\$ 40.00$ only $8.49 \%$ attended day-care center. For children with per capita income of US\$80.00 or more the proportion reaches $28.37 \%$. The lowest day-care center attendance was observed in the Midwest region (only $10.94 \%$ of the children had access to the service). The analysis of the nutritional status of children showed a higher proportion of children with $H A Z<-2$ among those not attending day-care center.
\end{abstract}

Index terms: anthropometry, child care, nutritional status, infant nutrition, health policy, child health.

\footnotetext{
(1) Pesquisa financiada pelo FNDCT - FINEP/BID.

(2) Departamento de Agroindústria, Alimentos e Nutrição, Escola Superior de Agricultura "Luiz de Queiroz", Universidade de São Paulo. Campus de Piracicaba, Caixa Postal 9, 13418-900, Piracicaba, SP, Brasil. Correspondência para/Correspondence to: M.V. SILVA.E-mail: mvdsilva@carpa.ciagri.usp.br
} 


\section{INTRODUÇÃO}

Por ser um período particularmente vulnerável, os primeiros anos de vida são decisivos para o crescimento das crianças.

Pesquisa de Monteiro (1984) revelou que o déficit estatural de crianças aos 7 anos já estava estabelecido aos 24 meses de idade.

Reconhece-se que, programas que tenham por base os cuidados infantis disponibilizados de forma integral e com boa qualidade, destinados às crianças, desde os primeiros meses de vida, podem exercer papel preventivo no sentido de protegê-las de distúrbios nutricionais, especialmente a desnutrição crônica.

Recentemente, tem sido observado o interesse de diversos autores (Fonseca et al., 1996; Fuchs et al., 1996; Silva, 1996; Silva \& Sturion, 1998) em avaliar o impacto da freqüência à creche sobre o estado nutricional das crianças.

Silva (1996) analisando dados relativos a crianças da faixa etária de 6 a 16 anos constatou a influência benéfica da freqüência a creche, nos primeiros anos de vida, no estado nutricional (efeito tardio) do grupamento de escolares observados, pertencentes as famílias de menor renda domiciliar per capita.

Os resultados da pesquisa de Silva \& Sturion (1998), tendo por base a análise do estado nutricional de 2096 crianças menores de 7 anos, informações socioeconômicas da família e tempo de freqüência a creche mostraram a influência estatisticamente significativa (teste de qui-quadrado, significativo a $1 \%$ ) exercida pelo tempo de permanência em creches sobre o escore- $Z$ de altura para a idade das crianças.

É importante frisar que, no Brasil, desde a sua criação no início do século XX até a década de 60 , as creches foram vistas como entidades assistenciais e, após 1979, tornaram-se um direito do trabalhador (Kramer, 1987; Vieira, 1988) ${ }^{3}$.

De acordo com Antonio et al. (1996), nos últimos 20 anos, houve um aumento significativo no número de creches no país, sem que se intensificasse a vigilância eficaz sobre as normas que regulamentam sua implantação e funcionamento.

Especificamente quanto ao estado nutricional das crianças brasileiras, no final da década de 80, destaca-se as análises de Monteiro (1992), que tendo por base os dados da Pesquisa Nacional sobre Saúde e Nutrição (PNSN) (Instituto Nacional..., 1990) revelou que a prevalência da desnutrição crônica atinge 15,4\% das crianças menores de cinco anos, observando, ainda, que a distribuição regional da desnutrição não era homogênea, sendo significativamente maior nos estados do Norte urbano e Nordeste.
A análise dos dados da PNSN, agrupados por unidades da federação e setor, permitiu a Hoffmann (1995) verificar que o percentual de crianças com déficits de altura é especialmente maior na zona rural de praticamente todos os estados brasileiros.

No entanto, Peliano (1990), observou que, de modo geral, a cobertura dos programas dirigidos às crianças, como é o caso da suplementação alimentar, é maior nas regiões mais desenvolvidas, nas áreas urbanas e entre crianças oriundas de famílias com maior poder aquisitivo.

Tanner (1978) revelou que as crianças do setor rural são freqüentemente menores e apresentam ritmo de crescimento mais lento do que as que vivem em áreas urbanas, em decorrência de alguns fatores tais como, a maior precariedade dos serviços de saúde e educação e a irregularidade do consumo de alimentos. O autor destaca, também, que as crianças das áreas rurais despendem mais energia em atividades físicas e que freqüentemente o atendimento da necessidade energética é menor do que o apresentado por crianças do setor urbano.

Ainda com base nos dados individuais da PNSN, Kassouf (1996) concluiu que, o fato da mãe trabalhar contribui para o aumento da renda familiar, no entanto, repercute negativamente sobre a saúde da criança, pois o tempo destinado para cuidar da criança e amamentá-la diminui. Uma alternativa para mães de crianças muito pequenas, que optam por participar do mercado de trabalho, é dispor de creches e outros programas substitutos dos cuidados maternos.

Indiscutivelmente é amplo o acervo de análises, sobre a situação nutricional das crianças brasileiras, especialmente às relativas aos dados obtidos no final da década de 80. No entanto, transcorridos 10 anos da realização da PNSN, são reduzidas as análises que exploram as informações sobre o acesso das crianças às creches.

Face ao exposto, optou-se por descrever, tendo por base os dados individuais da PNSN, o acesso à creche das crianças menores de 6 anos, segundo idade, estado nutricional, macro-regiões e estratos de renda a que pertencem.

\section{CASUÍSTICA E MÉTODOS}

\section{Base de dados utilizados}

Foram utilizados como base de dados as informações da Pesquisa Nacional sobre Saúde e Nutrição (Instituto Nacional..., 1990).

A amostra da referida pesquisa abrangeu cinco grandes regiões socioeconômicas e os setores urbano e rural do Brasil, exceto o norte rural. Na sua estrutura, a

\footnotetext{
${ }^{(3)}$ O artigo 208, inciso VI, do capítulo VIII (Da Educação, da Cultura e do Desporto), ao definir que o "dever do Estado com a educação será efetivado mediante garantia de (...) atendimento em creche e pré-escola às crianças de zero a seis anos de idade"; e no artigo $7^{\circ}$, inciso XXV, do Capítulo II (Dos Direitos Sociais) quando inclui, entre outros direitos dos trabalhadores urbanos e rurais "assistência gratuita aos filhos e dependentes desde o nascimento até seis anos de idade em creches e pré-escolas." Deve-se atentar, também, para o Estatuto da Criança e do Adolescente (capítulo IV, artigo 54) de 1990 e a Lei de Diretrizes e Bases de 1996 (artigo 4, inciso IV "atendimento gratuito em creches e pré-escolas às crianças de zero a seis anos de idade".
} 
amostra incluiu a investigação de 17920 domicílios, encontrados em 486 setores censitários. A população pesquisada foi de 63213 pessoas de todas as idades, residentes em 363 municípios brasileiros.

Este trabalho restringe-se à análise dos dados referentes à amostra de crianças com idade inferior a 6 anos $(n=10667)$.

\section{Estado nutricional}

A aferição do estado nutricional é, na PNSN, realizada pela adoção de medidas antropométricas que relacionam o peso e a altura das crianças amostradas com a população de referência recomendado pela Organização Mundial de Saúde (World..., 1995). Assim, dada a estatura/ altura ou o peso de uma criança calcula-se o valor do escore-Z que corresponde ao número de desvios-padrão abaixo ou acima da mediana da população de referência.

Neste trabalho, utilizou-se os valores individuais dos escores- $Z$ de altura para idade $(\mathrm{ZAI})$, peso para a altura (ZPA) e peso para idade (ZPI).

Foram definidos três intervalos de valores de Z: menor que -2 , de -2 a menos que -1 e pelo menos igual a -1 .

Em uma população com boas condições de saúde e nutrição, aproximadamente, $2,3 \%{ }^{4}$ dos valores estarão no primeiro intervalo, $13,6 \%$ estarão no segundo intervalo e os demais $84,1 \%$ deverão ter escore- $Z \geq-1$ (Silva, 1998).

Neste trabalho a proporção de crianças com escore ZAI <-2 será utilizada como indicador de desnutrição crônica e a proporção com ZAI $\geq-1$, indicador de eutrofia.
A situação de peso para a altura (ZPA) menor que - 2 aponta para o déficit de peso (desnutrição aguda) e o escore-Z de peso para idade (ZPI), por sua vez, é uma medida mista que reflete, parcialmente, as duas formas de desnutrição.

\section{Região do país e estratos de renda}

As crianças foram estratificadas de acordo com os conjuntos formados pelas macro-regiões do Brasil: Norte e Nordeste, Sul e Sudeste e Centro-Oeste.

Distinguiram-se três intervalos de estratos de renda domiciliar per capita: $\leq$ US\$40, US\$40-80 e $\geq$ US\$80.

Elaborou-se, também, a estratificação das crianças de acordo com a freqüência às creches que forneciam refeições gratuitas.

\section{RESULTADOS E DISCUSSÃO}

Os dados permitem verificar que, entre o grupo de crianças menores de dois anos de idade, apenas 3,6\% freqüentavam creche. No entanto, a proporção de crianças mais velhas (idade acima de cinco anos) com acesso à creche alcança 28,22\% (Tabela 1). Os resultados revelam uma diferença bastante acentuada, indicando que substancial parcela das crianças, consideradas biologicamente mais vulneráveis, são excluídas desse tipo de benefício.

Tabela 1. Distribuição das crianças de acordo com faixas de idade e acesso à creche. Brasil, 1989.

\begin{tabular}{|c|c|c|c|c|c|c|}
\hline \multirow{3}{*}{$\begin{array}{l}\text { Faixas de idade } \\
\text { (em anos) }\end{array}$} & \multicolumn{4}{|c|}{ Acesso à creche } & \multirow{2}{*}{\multicolumn{2}{|c|}{ Total }} \\
\hline & \multicolumn{2}{|c|}{ Não Freqüenta } & \multicolumn{2}{|c|}{ Freqüenta } & & \\
\hline & $\mathrm{n}$ & $\%$ & $\mathrm{n}$ & $\%$ & $\mathrm{n}$ & $\%$ \\
\hline$<2$ & 5791 & 96,40 & 216 & 3,60 & 6007 & 56,31 \\
\hline $2 \longmapsto 5$ & 2321 & 76,42 & 716 & 23,58 & 3037 & 28,47 \\
\hline $5 \vdash 6$ & 1165 & 71,78 & 458 & 28,22 & 1623 & 15,22 \\
\hline Total & 9277 & 86,97 & 1390 & 13,03 & 10667 & 100,00 \\
\hline
\end{tabular}

Tabela 2. Distribuição das crianças de acordo com as classes de renda domiciliar per capita e o acesso à creche. Brasil, 1989.

\begin{tabular}{|c|c|c|c|c|c|c|}
\hline \multirow{3}{*}{$\begin{array}{l}\text { Classes de renda } \\
\text { (em dólares) }\end{array}$} & \multicolumn{4}{|c|}{ Acesso à creche } & \multirow{2}{*}{\multicolumn{2}{|c|}{ Total }} \\
\hline & \multicolumn{2}{|c|}{ Não Freqüenta } & \multicolumn{2}{|c|}{ Freqüenta } & & \\
\hline & $\mathrm{n}$ & $\%$ & $\mathrm{n}$ & $\%$ & $\mathrm{n}$ & $\%$ \\
\hline$<40$ & 6061 & 91,51 & 558 & 8,49 & 6574 & 63,71 \\
\hline $40 \vdash 80$ & 1629 & 84,93 & 289 & 15,07 & 1918 & 18,59 \\
\hline$\geq 80$ & 1308 & 71,63 & 518 & 28,37 & 1826 & 17,70 \\
\hline Total & 8953 & 86,77 & 1365 & 12,23 & 10318 & 100,00 \\
\hline
\end{tabular}

\footnotetext{
(4) Note-se que 2,3\% corresponde à freqüência de crianças geneticamente baixas e/ou magras, observadas em população considerada de referência.
} 
Ao examinar os resultados das análises envolvendo o acesso à creche de acordo com as classes de renda domiciliar per capita, constata-se novamente o fenômeno da disparidade. Note-se que, entre as crianças de menor renda (<US\$40.00), 91,57\% não freqüentavam creche, enquanto entre crianças da classe de maior renda ( $\geq$ US\$80.00) a proporção alcança 71,63\% (Tabela 2).

A comparação entre as regiões mostra que a menor cobertura $(10,94 \%)$ das creches é verificada na Região Centro-Oeste (Tabela 3). É interessante registrar que análises elaboradas por Ometto et al. (1999) revelaram que no setor urbano da Região Centro-Oeste foi encontrada proporção de 6,39 crianças desnutridas, entre as que freqüentavam creche. No entanto esse valor é aproximadamente um terço do verificado no setor urbano da Região Sul/Sudeste (21,57\%).

Ainda de acordo com Ometto et al. (1999), é invariavelmente menor o percentual de crianças do setor rural de todas as regiões do país que freqüentavam creches.

A despeito dessa análise, cabe registrar a maior dificuldade da implementação de programas de creches na área rural devido a menor densidade populacional. Além disso, pode-se supor que a maior facilidade de conciliação do trabalho feminino na agricultura com o cuidado das crianças desestimule as iniciativas de implementação desse serviço. Uma alternativa viável poderia ser a capacitação de agentes comunitários para atuar em creches domiciliares disseminadas pelas áreas rurais, descentralizando esse tipo de serviço.
Ao examinar o conjunto das três tabelas, observa-se que o principal problema das crianças é a desnutrição crônica, pois enquanto $16,13 \%$ do total de crianças analisadas apresentam déficits de altura, apenas 1,90 (proporção inferior à esperada), registram ZPA <-2 e, $7,31 \%$ de crianças apresentam ZPI $<-2$. O percentual de $7,31 \%$, embora expressivo, é também substancialmente menor aos valores verificados quando se considera a classificação das crianças de acordo com o indicador de altura para idade (Tabelas 4, 5 e 6). Considerando as informações contidas na Tabela 4, verifica-se que 16,67\% das crianças que não freqüentam creche revelam déficits de altura (ZAI <-2), enquanto no grupamento com acesso a esse tipo de serviço, o percentual é menor $(12,56 \%)$.

Ao examinar os resultados da Tabela 5, depreende-se que em ambos os grupos de crianças (com ou sem acesso à creche) a proporção de crianças com ZPA <-2 é inferior à observada em população adotada como referência. A mesma situação, relativamente favorável, é observada quando se analisa os dados da Tabela 6 .

Note-se que as análises dos escores ZPI (medida mista que reflete, parcialmente as duas formas de desnutrição) apontam para 7,59\% de crianças com escore ZPI <-2 entre o grupo que não freqüenta a creche, enquanto o fenômeno é observado em 5,43\% das crianças que freqüentam a creche.

Tendo em vista que o principal problema nutricional verificado entre as crianças brasileiras, examinadas em 1989, é a desnutrição crônica e,

Tabela 3. Distribuição das crianças de acordo com o acesso à creche e grupo de regiões. Brasil, 1989.

\begin{tabular}{|c|c|c|c|c|c|c|c|c|}
\hline \multirow{3}{*}{ Acesso à creche } & \multicolumn{6}{|c|}{ Regiões } & \multirow{2}{*}{\multicolumn{2}{|c|}{ Total }} \\
\hline & \multicolumn{2}{|c|}{ Norte e Nordeste } & \multicolumn{2}{|c|}{ Sul e Sudeste } & \multicolumn{2}{|c|}{ Centro-Oeste } & & \\
\hline & $\mathrm{n}$ & $\%$ & $\mathrm{n}$ & $\%$ & $\mathrm{n}$ & $\%$ & $\mathrm{n}$ & $\%$ \\
\hline Não freqüenta & 3787 & 84,68 & 3594 & 89,34 & 1896 & 89,06 & 9277 & 86,97 \\
\hline Freqüenta & 685 & 15,32 & 472 & 11,61 & 233 & 10,94 & 1390 & 13,03 \\
\hline \multirow[t]{2}{*}{ Total } & 4472 & 100,00 & 4066 & 100,00 & 2129 & 100,00 & 10667 & 100,00 \\
\hline & & {$[41,92]$} & & {$[38,12]$} & & {$[19,96]$} & & \\
\hline
\end{tabular}

$\chi^{2}=36,07$, com dois graus de liberdade significativo a $1 \%$

Obs.: Os números entre colchetes são os percentuais em relação ao total de crianças $(n=10667)$

Tabela 4. Distribuição das crianças de acordo com o acesso e o escore-Z de altura para idade (ZAI). Brasil, 1989.

\begin{tabular}{|c|c|c|c|c|c|c|c|c|}
\hline \multirow{3}{*}{ Acesso à creche } & \multirow{2}{*}{\multicolumn{2}{|c|}{ Crianças }} & \multicolumn{6}{|c|}{ Categorias do estado nutricional } \\
\hline & & & \multicolumn{2}{|c|}{$\mathrm{ZAI}<-2$} & \multicolumn{2}{|c|}{$-2 \leq \mathrm{ZAI}<-1$} & \multicolumn{2}{|c|}{$\mathrm{ZAI} \geq-1$} \\
\hline & $\mathrm{n}$ & $\%$ & $\mathrm{n}$ & $\%$ & $n$ & $\%$ & $\mathrm{n}$ & $\%$ \\
\hline Não freqüenta & 9160 & 86,93 & 1527 & 16,67 & 2295 & 15,05 & 5338 & 58,28 \\
\hline Freqüenta & 1377 & 13,07 & 173 & 12,56 & 299 & 21,71 & 905 & 65,72 \\
\hline Total & 10537 & 100,00 & 1700 & 16,13 & 2594 & 24,62 & 6243 & 59,25 \\
\hline
\end{tabular}

$\chi^{2}=29,15$, com dois graus de liberdade significativo a $1 \%$ 
também, o reconhecimento da importância da alimentação adequada como fator decisivo para o crescimento infantil, julgou-se pertinente elaborar as análises envolvendo a disponibilidade de refeições nas creches, de acordo com as macro-regiões onde essas se localizam e a situação nutricional das crianças, adotando-se o escore-Z de altura para idade (ZAI).

As Tabelas 7, 8 e 9, oferecem um panorama da situação, no final da década de 80. Chama a atenção o fato de que, em todas as regiões, de forma sistemática, maiores proporções de crianças com escore ZAI <-2 (indicativo de desnutrição crônica) freqüentam creches onde recebem refeições gratuitas. O maior diferencial é observado na Região Sul e Sudeste, onde 9,89\% das crianças com comprometimento da altura freqüentam creches com disponibilidade de refeições, enquanto $2,46 \%$ (valor quase 4 vezes menor) não tem acesso ao programa alimentar.

Tabela 5. Distribuição das crianças de acordo com o acesso à creche e o escore-Z de peso para a altura (ZPA). Brasil, 1989.

\begin{tabular}{|c|c|c|c|c|c|c|c|c|}
\hline \multirow{3}{*}{ Acesso à creche } & \multirow{2}{*}{\multicolumn{2}{|c|}{ Crianças }} & \multicolumn{6}{|c|}{ Categorias do estado nutricional } \\
\hline & & & \multicolumn{2}{|c|}{$\mathrm{ZPA}<-2$} & \multicolumn{2}{|c|}{$-2 \leq \mathrm{ZPA}<-1$} & \multicolumn{2}{|c|}{$\mathrm{ZPA} \geq-1$} \\
\hline & $\mathrm{n}$ & $\%$ & $\mathrm{n}$ & $\%$ & $\mathrm{n}$ & $\%$ & $\mathrm{n}$ & $\%$ \\
\hline Não freqüenta & 9097 & 86,90 & 181 & 1,99 & 906 & 9,96 & 8010 & 88,05 \\
\hline Freqüenta & 1371 & 13,10 & 18 & 1,31 & 114 & 8,32 & 1239 & 90,37 \\
\hline Total & 10468 & 100,00 & 199 & 1,90 & 1029 & 9,74 & 9249 & 88,35 \\
\hline
\end{tabular}

$\chi^{2}=6,90$, com dois graus de liberdade significativo a $1 \%$

Tabela 6. Distribuição das crianças de acordo com o acesso à creche e o escore -Z de peso para idade (ZPI). Brasil, 1989.

\begin{tabular}{|c|c|c|c|c|c|c|c|c|}
\hline \multirow{3}{*}{ Acesso à creche } & \multirow{2}{*}{\multicolumn{2}{|c|}{ Crianças }} & \multicolumn{6}{|c|}{ Categorias do estado nutricional } \\
\hline & & & \multicolumn{2}{|c|}{$\mathrm{ZPI}<-2$} & \multicolumn{2}{|c|}{$-2 \leq \mathrm{ZPI}<-1$} & \multicolumn{2}{|c|}{$\mathrm{ZPI} \geq-1$} \\
\hline & $\mathrm{n}$ & $\%$ & $\mathrm{n}$ & $\%$ & $\mathrm{n}$ & $\%$ & $\mathrm{n}$ & $\%$ \\
\hline Não freqüência & 9236 & 86,98 & 701 & 7,59 & 2204 & 23,86 & 6331 & 68,55 \\
\hline Freqüência & 1382 & 13,02 & 75 & 5,43 & 281 & 20,33 & 1026 & 74,24 \\
\hline Total & 10618 & 100,00 & 776 & 7,31 & 2485 & 23,40 & 7357 & 69,29 \\
\hline
\end{tabular}

$\chi^{2}=19,72$, com dois graus de liberdade significativo a $1 \%$

Tabela 7. Distribuição das crianças das Regiões Norte e Nordeste de acordo com a disponibilidade de refeições na creche e o estado nutricional. Brasil, 1989.

\begin{tabular}{|c|c|c|c|c|c|c|c|c|}
\hline \multirow{3}{*}{$\begin{array}{l}\text { Disponibilidade } \\
\text { de refeições }\end{array}$} & & & \multicolumn{6}{|c|}{ Categorias do estado nutricional } \\
\hline & \multicolumn{2}{|c|}{ Crianças } & \multicolumn{2}{|c|}{$\mathrm{ZAI}<-2$} & \multicolumn{2}{|c|}{$-2 \leq \mathrm{ZAI}<-1$} & \multicolumn{2}{|c|}{$\mathrm{ZAI} \geq-1$} \\
\hline & $\mathrm{n}$ & $\%$ & $\mathrm{n}$ & $\%$ & $\mathrm{n}$ & $\%$ & $\mathrm{n}$ & $\%$ \\
\hline Não recebe & 345 & 50,88 & 38 & 11,01 & 73 & 21,16 & 234 & 67,83 \\
\hline recebe & 333 & 49,12 & 92 & 27,63 & 112 & 33,63 & 129 & 38,74 \\
\hline Total & 678 & 100,00 & 130 & 19,17 & 185 & 27,29 & 363 & 53,54 \\
\hline
\end{tabular}

$\chi^{2}=60,83$, com dois graus de liberdade significativo a $1 \%$

Tabela 8. Distribuição das crianças das Regiões Sul e Suldeste de acordo com a disponibilidade de refeições na creche e o estado nutricional. Brasil, 1989.

\begin{tabular}{|c|c|c|c|c|c|c|c|c|}
\hline \multirow{3}{*}{$\begin{array}{l}\text { Disponibilidade } \\
\text { de refeições }\end{array}$} & & & \multicolumn{6}{|c|}{ Categorias do estado nutricional } \\
\hline & \multicolumn{2}{|c|}{ Crianças } & \multicolumn{2}{|c|}{$\mathrm{ZAI}<-2$} & \multicolumn{2}{|c|}{$-2 \leq \mathrm{ZAI}<-1$} & \multicolumn{2}{|c|}{$\mathrm{ZAI} \geq-1$} \\
\hline & $\mathrm{n}$ & $\%$ & $\mathrm{n}$ & $\%$ & $\mathrm{n}$ & $\%$ & $\mathrm{n}$ & $\%$ \\
\hline Não recebe & 203 & 43,56 & 5 & 2,46 & 20 & 9,85 & 178 & 87,68 \\
\hline Recebe & 263 & 56,44 & 26 & 9,89 & 48 & 18,25 & 189 & 71,86 \\
\hline Total & 466 & 100,00 & 31 & 6,65 & 68 & 14,59 & 367 & 78,76 \\
\hline
\end{tabular}

$\chi^{2}=18,67$, com dois graus de liberdade significativo a $1 \%$ 
Tabela 9. Distribuição das crianças da Região Centro-Oeste de acordo com a disponibilidade de refeições na creche e o estado nutricional. Brasil, 1989.

\begin{tabular}{|c|c|c|c|c|c|c|c|c|}
\hline \multirow{3}{*}{$\begin{array}{l}\text { Disponibilidade } \\
\text { de refeições }\end{array}$} & \multirow{2}{*}{\multicolumn{2}{|c|}{ Crianças }} & \multicolumn{6}{|c|}{ Categorias do estado nutricional } \\
\hline & & & \multicolumn{2}{|c|}{$\mathrm{ZAI}<-2$} & \multicolumn{2}{|c|}{$-2 \leq \mathrm{ZAI}<-1$} & \multicolumn{2}{|c|}{$\mathrm{ZAI} \geq-1$} \\
\hline & $\mathrm{n}$ & $\%$ & $\mathrm{n}$ & $\%$ & $\mathrm{n}$ & $\%$ & $\mathrm{n}$ & $\%$ \\
\hline Não recebe & 124 & 53,45 & 5 & 4,03 & 22 & 17,74 & 97 & 78,23 \\
\hline Recebe & 108 & 46,55 & 7 & 6,48 & 24 & 22,22 & 77 & 71,30 \\
\hline Total & 232 & 100,00 & 12 & 5,17 & 46 & 19,83 & 174 & 75,00 \\
\hline
\end{tabular}

$\chi^{2}=1,62$, com dois graus de liberdade, não-significativo

Ao examinar as informações da Tabela 7, é interessante observar que, quando se considera o conjunto ( $n=130 ; 19,17 \%$ ) de crianças com indicativo de déficit de altura (escore ZAI <-2), nota-se que $11,01 \%(n=38)$, freqüentavam no final da década de 80 , creches que não ofereciam refeições gratuitas.

Ainda de acordo com a Tabela 7, vale destacar a situação, também desfavorável de praticamente $64 \%$ $(n=234)$ das crianças eutróficas que, embora freqüentando creches, não têm acesso às refeições. É possível que essa condição tenha contribuído de forma negativa, particularmente, para a situação nutricional das crianças, pertencentes às regiões mais pobres do Brasil.

\section{CONCLUSÃO}

Foi possível verificar que irrisória parcela das crianças de menor idade, considerada de maior vulnerabilidade biológica, tinha em 1989, acesso às creches. Do mesmo modo, quando se analisou o acesso do grupamento mais pobre (<US\$40.00 per capita), constatou-se que aproximadamente $90,0 \%$ não freqüentavam creches.

Os resultados obtidos revelam que, no final da década (considerada perdida pelos economistas) era notória a necessidade de priorizar o atendimento, de forma bastante precoce, das crianças e, principalmente, ampliar a cobertura dos serviços, visando alcançar, com prioridade, o grupo de crianças pertencentes às famílias de menor renda.

No tocante a distribuição regional, o estado nutricional e a freqüência a creche com disponibilidade de refeições, os resultados mostraram que esse tipo de atendimento alcançava as crianças com déficits de altura (desnutridos crônicos).

No entanto é pertinente lembrar que invariavelmente o déficit estatural estabelece-se durante os dois primeiros anos de vida e portanto, todos os esforços deveriam ser orientados no sentido de oferecer às crianças de menor idade acesso à assistência à saúde, condições de infra-estrutura domiciliar, e, na medida do possível, orientações às mães visando a ampliação do período de aleitamento materno e, também, maior capacitação para os cuidados infantis.

$\mathrm{O}$ acesso à creche e o acompanhamento das crianças, especialmente as que apresentam distúrbios nutricionais, devem ser assegurados.

Embora seja indiscutível a relevância de políticas sociais visando amenizar os graves problemas que afetam a população brasileira, fica clara a necessidade de uma reorientação no sentido de direcioná-las para os grupos, tradicionalmente fora do alcance das políticas públicas.

Espera-se que novos trabalhos, baseados em pesquisas realizadas na década de 90 possibilitem, entre outras, a construção de análises temporais relativas ao acesso das crianças brasileiras aos programas de creches.

Espera-se, também, que uma nova pesquisa, nos moldes da PNSN, que inclua informações sobre a qualidade dos serviços, oferecidos pelas creches, seja implementada, com a maior brevidade.

\section{REFERÊNCIAS BIBLIOGRÁFICAS}

ANTONIO, M.A.G.M., MORCILLO, A.M., PIEDRABUENA, A.E., CARNIEL, E.F. Avaliação nutricional das crianças matriculadas nas quatorze creches municipais de Paulínia, SP. Revista Paulista de Pediatria, São Paulo, v.14, n.1, p.10-16, 1996.

FONSECA, W., KIKWOOD, B.R., BARROS, A.J.D., MISAGO, C., CORREIA, L.L., FLORES, J.A.M., FUCHS, S.C., VICTORIA, C.G. Attendance at day care centers increases the risk of childhood pneumonia among the urban poor in Fortaleza, Brazil. Cadernos de Saúde Pública, Rio de Janeiro, v.12, n.2, p.133-140, 1996.

FUCHS, S.C., MAYNART, R.C., COSTA, L.F., CARDOSO, A., SCHIERHOLT, R. Duration of day care attendance and acute respiratory infection. Cadernos de Saúde Pública, Rio de Janeiro, v.12, n.3, p.291-296, 1996.

HOFFMANN, R. Pobreza e desnutrição de crianças no Brasil: diferenças regionais e setoriais. In: ENCONTRO NACIONAL DE ECONOMIA, 23., 1995, Salvador. Anais... São Paulo : Associação Nacional de Pós-Graduação em Economia, 1995. v.2: p.442-453. 
INSTITUTO NACIONAL DE ALIMENTAÇÃO E NUTRIÇÃO. Pesquisa Nacional sobre Saúde e Nutrição. Brasília, 1990. (Dados individuais disponíveis em meio magnético).

KASSOUF, A.L. Saúde e nutrição de crianças nos setores urbanos e rural. In: TEIXEIRA, E.C., VIEIRA, W.C. (Org.). Reforma da política agrícola e abertura econômica. Viçosa : UFV, 1996. 210p.

KRAMER, S.A. A política do pré-escolar no Brasil: a arte do disfarce. 3.ed. Rio de Janeiro : Achiamé, 1987. 131p.

MONTEIRO, C.A. Critérios antropométricos no diagnóstico da desnutrição em programas de assistência à criança. Revista de Saúde Pública, São Paulo, v.18, n.3, p.209-217, 1984.

MONTEIRO, C.A. Saúde e nutrição das crianças brasileiras no final da década de 80. In: PERFIL estatístico de crianças e mães no Brasil: aspectos de saúde e nutrição de crianças no Brasil, 1989. Rio de Janeiro, 1992. 129 p.

OMETTO, A.M.H., SILVA, M.V., FURTUOSO, M.C.O., OETTERER, M., PIPITONE, M.A.P., STURION, G.L. O estado nutricional de crianças brasileiras: o efeito de programas de suplementação alimentar, saúde e saneamento. In: OETTERER, M., SILVA, M.V., OMETTO, A.M.H., PIPITONE, M.A.P., FURTUOSO, M.C.O., STURION, G.L. (Org.). Avaliação do programa de alimentação escolar. Piracicaba, 1999. 365p. (Relatório final de pesquisa).

PELIANO, A.M. Quem se beneficia dos programas governamentais de suplementação alimentar. Núcleo de
Pesquisa em Políticas Públicas. Campinas : Unicamp, 1990. 26p. (Texto para discussão, n.205).

SILVA, M.V. Estado nutricional de escolares matriculados em Centros Integrados de Educação Pública - CIEP's. São Paulo, 1996. 110p. Tese (Doutorado em Saúde Pública) - Faculdade de Saúde Pública, Universidade de São Paulo, 1996.

SILVA, M.V. Estado nutricional de alunos matriculados em escolas públicas de tempo integral. Archivos Latinoamericanos de Nutrición, Guatemala, v.48, n.1, p.18-24, 1998.

SILVA, M.V., STURION, G.L. Freqüência à creche e outros condicionantes do estado nutricional infantil. Revista de Nutrição, Campinas, v.11, n.1, p.58-68, 1998.

TANNER, J.M. Foetus into man: physical growth from conception to maturity. Massachusetts : Harvard University Press, 1978. $250 \mathrm{p}$.

VIEIRA, L.M.F. Mal necessário: creches no Departamento Nacional da Criança (1940-1970). Cadernos de Pesquisas, São Paulo, v.67, p.3-16, 1988. (Volume Único).

WORLD HEALTH ORGANIZATION. The use and interpretation of anthropometry. Geneva, 1995. 452p. (Technical Report Series, n.854).

Recebido para publicação em 13 de agosto e aceito em 23 de novembro de 1999. 